\title{
Assistência de enfermagem a mulher em período puerperal: uma revisão integrativa
}

\author{
Nursing assistance to women in puerperal period: an integrative review
}

Asistencia de enfermería a la mujer en período puerperal: una revisión integradora

Recebido: 02/11/2021 | Revisado: 07/11/2021 | Aceito: 10/11/2021 | Publicado: 14/11/2021

\author{
Dean Douglas Ferreira de Olivindo \\ ORCID: https://orcid.org/0000-0002-9433-2625 \\ Centro Universitário Santo Agostinho, Brasil \\ E-mail: dean_olivindo@yahoo.com.br \\ Lara Pinheiro Costa \\ ORCID: https://orcid.org/0000-0002-1426-1223 \\ Centro Universitário Santo Agostinho, Brasil \\ E-mail: lara20900@outlook.com \\ Thais Bell Barbosa de Moraes Trindade \\ ORCID: https://orcid.org/0000-0002-0650-052X \\ Centro Universitário Santo Agostinho, Brasil \\ E-mail: thaisbelabell@gmail.com \\ Thamires Barbosa dos Santos \\ ORCID: https://orcid.org/0000-0002-6076-5473 \\ Faculdade Adelmar Rosado, Brasil \\ E-mail: thamireesfisio@gmail.com
}

\begin{abstract}
Resumo
Objetivou-se identificar e descrever as evidências científicas de enfermagem a mulheres em período puerperal. Tratase de uma revisão integrativa da literatura. A realização do estudo ocorreu através de consulta indireta de produções cientificas disponível na plataforma da Biblioteca Virtual em Saúde (BVS) nas seguintes bases de dados on-line Literatura Latino Americana em Ciências da Saúde (LILACS), Banco de Dados em Enfermagem (BDENF) e Medical Literature Analysis and Retrieval System Online (MEDLINE) com recorte temporal de 2016 a 2021. Foram utilizados os descritores "Assistência de Enfermagem"; "Puerpério"; "Saúde da mulher". O estudo teve como critérios de Inclusão: artigos em Português, Inglês ou Espanhol, artigos originais na íntegra, disponíveis e gratuitas. Estabeleceram-se como critérios de exclusão artigos que não atendiam aos objetivos da pesquisa, estudos de revisão e resumos. Foram encontrados 219 estudos, após aplicação dos critérios de inclusão onde foram selecionados 75 artigos para leitura dos resumos, logo após foram selecionados para fazer parte do estudo 10 artigos que atenderam aos objetivos da pesquisa. Após análise dos resultados surgiram duas categorias para discussão da temática, sendo elas: Assistência de enfermagem do puerpério; A percepção da puérpera sobre a assistência de enfermagem. Conclui-se que a enfermagem deva estar constantemente se aprimorando para uma assistência que possa atender as demais demandas e singularidades de cada puérpera, pois o puerpério é uma fase com possibilidade de riscos e agravos, onde os cuidados devem visar à prevenção de complicações na mãe e também no recém-nascido.
\end{abstract}

Palavras-chave: Assistência de enfermagem; Puerpério; Saúde da mulher.

\begin{abstract}
The objective was to identify and describe the scientific evidence of nursing to women in the puerperal period. This is an integrative literature review. The study was carried out through indirect consultation of scientific productions available on the Virtual Health Library (VHL) platform in the following online databases Latin American Literature in Health Sciences (LILACS), Database in Nursing (BDENF) and Medical Literature Analysis and Retrieval System Online (MEDLINE) with a time frame from 2016 to 2021. The descriptors "Nursing Care" were used; "Puerperium"; "Women's Health". The study had as inclusion criteria: articles in Portuguese, English or Spanish, original articles in full, available and free of charge. Exclusion criteria were established articles that did not meet the research objectives, review studies and abstracts.219 studies were found, after applying the inclusion criteria where 75 articles were selected for reading the abstracts, then 10 articles that met the research objectives were selected to be part of the study. After analysis of the results two categories emerged for discussion of the theme, namely: Postpartum nursing care; The puerperal woman's perception of nursing care. It is concluded that nursing must be constantly improving for assistance that can meet the other demands and singularities of every puerperal woman, because the puerperium is a phase with the possibility of risks and harm, where care must be aimed at prevention complications in the mother and also in the newborn.
\end{abstract}

Keywords: Nursing care; Postpartum; Women's health. 


\section{Resumen}

El objetivo fue describir e identificar la evidencia científica de la enfermería a la mujer en el puerperio. Se trata de una revisión integradora de la literatura, el estudio se llevó a cabo mediante investigación material mediante consulta directa de producciones científicas disponibles en la plataforma Biblioteca Virtual en Salud (BVS) en las siguientes bases de datos en línea Literatura Latinoamericana en Ciencias de la Salud (LILACS), Base de Datos de Enfermería (BDENF) y Sistema de análisis y recuperación de literatura médica en línea (MEDLINE). con marco de tiempo de 2016 a 2021. Se utilizaron los descriptores "Asistencia de enfermería", "Puerperio", "Salud de la mujer". El estudio tuvo como criterios de inclusión: artículos en portugués, inglés o español, artículos originales completos, disponibles y gratuitos. Se establecieron como criterios de exclusión artículos que no cumplieran con los objetivos de la investigación, estudios de revisión y resúmenes. Se encontraron un total de 219 estudios, luego de aplicar los criterios de inclusión donde se seleccionaron 75 artículos para la lectura de los resúmenes, luego se seleccionaron 10 artículos que cumplieron con los objetivos de esta investigación para formar parte del estudio. Luego de analizar los resultados, surgieron dos categorías para la discusión del tema, a saber: Atención de enfermería posparto; Percepción de la puérpera sobre los cuidados de enfermería. Se concluye que la enfermería debe estar mejorando constantemente para una asistencia que pueda satisfacer las demás demandas y singularidades de cada puérpera. El puerperio es una fase con posibilidad de riesgos y lesiones, donde los cuidados deben estar orientados a prevenir complicaciones en la madre y el recién nacido.

Palabras clave: Atención de enfermería; Posparto; La salud de la mujer.

\section{Introdução}

A experiência do puerpério é um momento único com transformações em que a mulher vivência mudanças e adaptações físicas e psíquicas, assumindo uma nova realidade na transição ao papel materno, principalmente quando é o primeiro filho. Este período tem uma pausa de seis semanas, mas pode se estender do nascimento do bebê até a normalização fisiológica, tornando-se capaz de ter uma extensão variável. Sendo assim pode ser dividido em três fases: puerpério imediato, que vai do $1^{\circ}$ até o $10^{\circ}$ dia do pós-parto; puerpério tardio, que prossegue do $11^{\circ}$ ao $42^{\circ}$ dia, e puerpério remoto, que continua do $43^{\circ}$ dia (Gonçalves et al., 2012; Cunha et al., 2014)

Ocorrem diversas modificações neste período, anatômicas e fisiológicas como a diminuição uterina, eliminação dos lóquios, trato urinário, modificações das mamas, entre vários outros. Podendo ocorrer também adaptações emocionais, que podem gerar na puérpera ansiedade, desconforto físico decorrente ao tipo de parto, inseguranças em relação à amamentação, o medo da incapacidade de cuidar e conseguir compensar às necessidades do bebê. Nesta fase a mulher precisa de apoio, e deve ser cercados de cuidados de qualidade e humanizados. Pois é dever dos profissionais principalmente a enfermagem que tem mais contato com o paciente de acolher com dignidade e respeito a mulher e o recém-nascido, enfocando-os como sujeitos dos seus direitos. (Pigrol \& Baruffi, 2017).

Ainda que a maior parte das alterações no puerpério seja fisiológica, as puérperas atravessam por medos, desafios, anseios e até mesmo de situações de risco que possam impactar negativamente obinômio mãe-filho. Somam-se a estes riscos os problemas reais já instalados, que apresentam a demanda da atuação da enfermagem por meio de uma assistência mais próxima da mulher. As mulheres se encontram nesta fase mais vulneráveis e sensíveis a receberem influências dos profissionais da saúde. Um dos critérios determinados pelo Ministério da Saúde para a qualidade da assistência ao pós-parto é o auxilio à mulher e ao recém-nascido durante o puerpério imediato e tardio.Consequentemente, os profissionais devem aproveitar a oportunidade desta fase na vida da mulher para execução de ações para a promoção da saúde e prevenção de doenças, tal como realizar a educação em saúde, buscando sanar duvidas que essas puérperas possam ter. (Souza \& Fernandes, 2014).

Segundo Brasil (2011) o decreto Presidencial n 1.459 de 24/06/2011 no âmbito do SUS, a rede cegonha, assegura à mulher o direto ao planejamento reprodutivo e atenção humanizada à gravidez, ao parto e ao puerpério (pós-parto) e, às crianças, o direito ao nascimento seguro e ao crescimento e desenvolvimento saudável. Sendo assim prevenindo situações de agravos e doenças durante o ciclo gravídico-puerperal. 
A assistência à mulher no pós-parto imediato e nas primeiras semanas após o parto é de fundamental importância para a saúde materna e neonatal. A enfermagem está diretamente ligada à realização da assistência puerperal através das consultas de enfermagem, e prestação no campo domiciliar, a mesma é executada ainda na primeira semana após o parto, onde possibilita a prestação de cuidados referentes à mulher e ao bebê, para prevenção das intercorrências da lactação e outras complicações do período. Os profissionais de enfermagem devem se atentar as necessidades que as puérperas podem ter principalmente física e mental, se colocando no lugar das puérperas par assim prestar uma assistência de qualidade e humanizado (Brasil, 2012).

O estudo se justifica através da importância de uma assistência de enfermagem de qualidade na saúde da mulher que vivência a fase do puerpério focando na importância dos cuidados a esse público que vivencia uma profunda e definitiva transformação, merecendo ser destacadas, já que maioria das vezes o cuidado é focado no recém-nascido em detrimento da mãe. Portanto este estudo busca contribuir para o conhecimento acadêmico e para os que trabalham na área da saúde, servindo de subsidio para novas pesquisas.

Sabendo da relevância do papel desempenhado pelo enfermeiro a fim de evitar problemas de saúde e intercorrências indesejáveis e visando compreender melhor essa abordagem, o estudo tem como objetivo: descrever e identificar as evidências científicas da assistência de enfermagem a mulheres em período puerperal.

\section{Metodologia}

Trata-se de uma pesquisa bibliográfica de abordagem qualitativa realizada pelo método revisão integrativa.Segundo Mendes, Silveira e Galvão (2008) a revisão integrativa inclui a análise de pesquisas relevantes que dão suporte para a tomada de decisão e a desenvolvimento da prática clínica, possibilitando a síntese do ponto do conhecimento de um determinado assunto, além de indicar lacunas do conhecimento que precisam ser preenchidas com a realização de novos estudos (Souza et al., 2010).

Este tipo de revisão apresenta-se em seis fases segundo Mendes, Silveira e Galvão (2008). 1- Elaboração da pergunta norteadora; 2- amostragem; 3- coleta de dados; 4- análise crítica dos estudos incluídos; 5- discussão de resultados; 6apresentação da revisão.

Para Dyniewicz (2014) O método qualitativo proporciona campo livre às percepções e subjetividades dos seres humanos através da descrição da experiência humanos tal como ela é vivida e tal como é definida pelos seus próprios autores.

Para a realização do estudo foram utilizadas como fonte de coleta as plataformas online de pesquisa, Biblioteca Virtual em Saúde (BVS) de forma indireta nas seguintes bases de dados: Literatura Latino Americana em Ciências da Saúde (LILACS), Banco de Dados em Enfermagem (BDENF) e Medical Literature Analysis and Retrieval System Online (MEDLINE), utilizando-se palavras chave que compõe parte dos Descritores em Ciência da Saúde (DeCS), foi realizado o cruzamento dos descritores acompanhado do operador booleano "AND": "Assistência de Enfermagem AND Puerpério AND Saúde da mulher", possibilitando a junção dos termos escolhidos. Para nortear a busca dos artigos de acordo com as normas da revisão integrativa foi elaborada a seguinte questão: “Qual a assistência do profissional enfermeiro a mulher em período de puerpério?".

Na segunda etapa o estudo teve como critérios de Inclusão: artigos em Português, Inglês ou Espanhol, por serem idiomas com maior quantidade de publicações, artigos originais na íntegra, disponíveis e gratuitos online, publicados nos últimos cinco anos, de 2016 a 2021 por abranger os estudos mais recentes, e que atendessem a temática definida. Estabeleceram-se como critérios de exclusão: artigos que não atendiam aos objetivos da pesquisa, estudos de revisão e 
resumos, publicações não disponíveis na integra e que não estivessem disponíveis online, estudos duplicados e estudos que não atendiam ao objetivo dessa pesquisa.

A terceira etapa se constituiu a coleta de dados, onde a análise do material ocorreu no mês de setembro de 2021. Através da busca de artigos a partir da BVS realizando o cruzamento dos descritores já citados acima resultou em 219 estudos encontrados através de pesquisa em banco de dados. Em seguida 75 artigos foram selecionados após filtragem, que passaram por uma analise a partir da leitura dos títulos e resumos, logo 57 estudos foram excluídos por não atenderem os critérios de inclusão, onde 18 foram analisados para avaliar elegibilidade, sendo 8 artigos excluídos por não atenderem os objetivos da pesquisa, por fim sendo selecionados para fazer parte do estudo 10 artigos que atenderam aos objetivos da presente pesquisa, conforme a Figura 1 apresenta.

A Quarta etapa que visa a análise crítica dos estudos incluídos na revisão, foi elaborado um quadro (Quadro 1), referente aos estudos obtidos como resultados na revisão integrativa contendo as distribuições dos artigos utilizados conforme título da obra, ano, autores e periódico, para sintetizar as informações extraídas dos artigos selecionados.

A quinta etapa visa à discussão de resultados através da exploração criteriosa das informações contidas nos estudos, com foco na questão norteadora. Nesta etapa ocorreu a discussão dos resultados obtidos e para isso, foi realizada uma leitura criteriosa de cada estudo para formular e extrair as informações e conceitos relevantes que incrementaram a pesquisa. E por fim, a sexta etapa que é apresentação da revisão/síntese do conhecimento para a construção da revisão integrativa. 
Figura 1: Fluxograma Metodológico-Etapas da pesquisa.
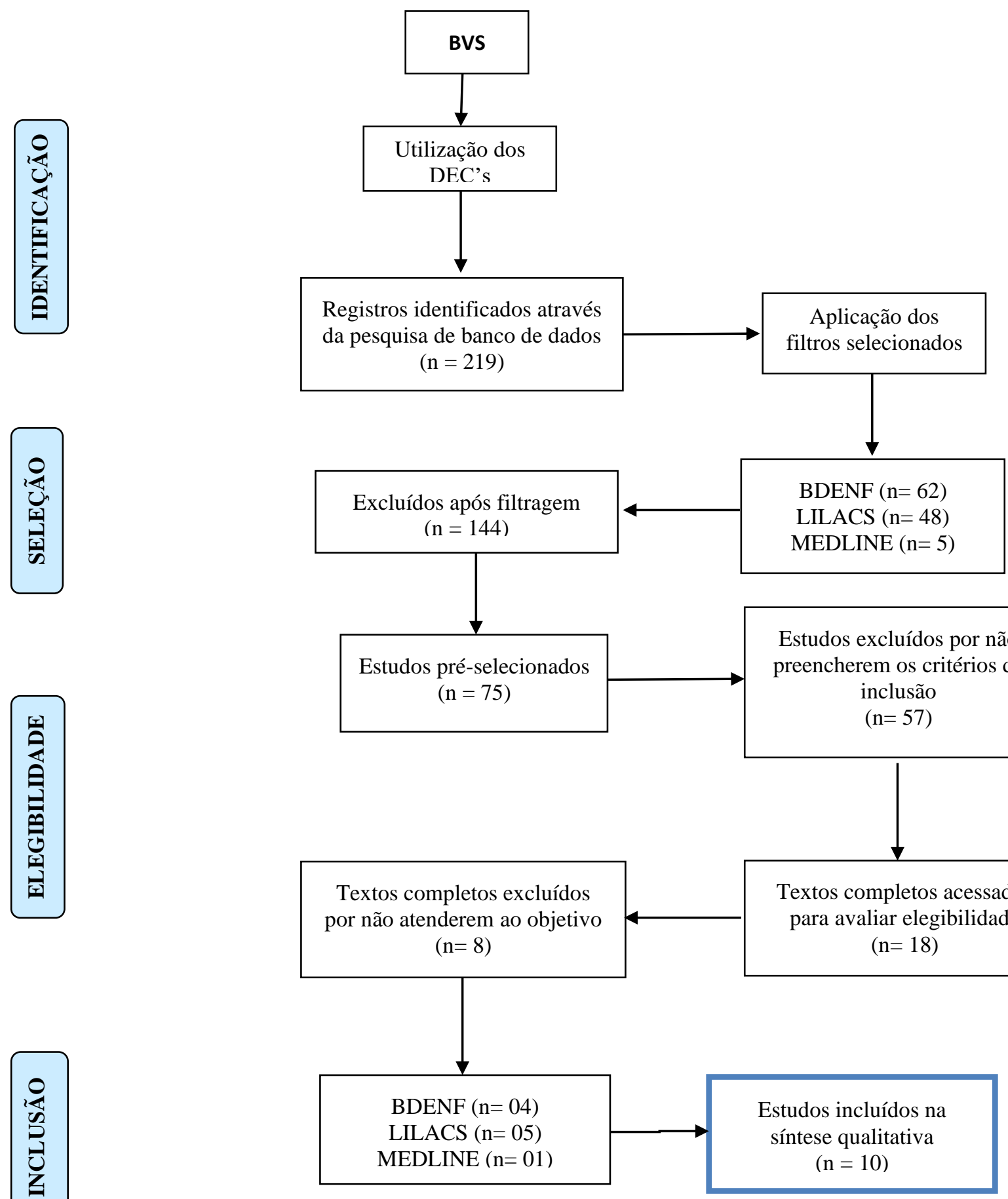

Fonte: Autores.

\section{Resultados}

A seguir, serão apresentadas as publicações científicas encontradas nas bases de dados LILACS, BDENF e MEDLINE após a seleção da amostra final, cujo qual na base de dados LILACS foram selecionados 05 estudos, na BDENF 04 
e na MEDLINE 01 estudo científico. Foram incluídos na síntese qualitativa 10 estudos, conforme descrições sintetizadas no Quadro 1.

Quadro 1. Distribuição dos artigos utilizados conforme título da obra, ano, autores e periódico.

\begin{tabular}{|c|c|c|c|}
\hline TÍTULO & ANO & AUTORES & PERIÓDICO \\
\hline $\begin{array}{c}\text { Proposta para a criação de uma consulta pós- } \\
\text { parto dada por Enfermagem Ginecologia, } \\
\text { Obstetrícia e Perinatal na Área de Saúde de } \\
\text { Coronado }\end{array}$ & 2016 & $\begin{array}{c}\text { Quirós, C. C.; Barboza } \\
\text { L. S }\end{array}$ & $\begin{array}{c}\text { Revista Electrónica } \\
\text { Enfermeria Actual en Costa } \\
\text { Rica }\end{array}$ \\
\hline $\begin{array}{l}\text { Percepções de puérperas sobre o cuidado de } \\
\text { enfermagem no alojamento conjunto }\end{array}$ & 2017 & Strefling, I. S. S.et al & $\begin{array}{l}\text { Rev. Pesqui. (Univ. Fed. } \\
\text { Estado Rio J., Online) }\end{array}$ \\
\hline O papel do Enfermeiro no cuidado à puérpera & 2017 & $\begin{array}{l}\text { Prigol, A. P.; Baruffi, } \\
\text { L.M }\end{array}$ & Rev. enferm. UFS \\
\hline $\begin{array}{l}\text { Puerpério e assistência de enfermagem: } \\
\text { percepção das mulheres }\end{array}$ & 2017 & Silva, E. C. et al & Rev. enferm. UFPE on line \\
\hline $\begin{array}{l}\text { Representações sociais de enfermeiros da } \\
\text { atenção primária à saúde sobre cuidado de } \\
\text { enfermagem no pós-parto }\end{array}$ & 2018 & Dantas, S. L.C. et al & Cogitare enfermagem \\
\hline $\begin{array}{l}\text { Compreensões de cuidado na visão de } \\
\text { mulheres puérperas }\end{array}$ & 2018 & Ebling, S. B. D.et al & $\begin{array}{l}\text { Rev. Pesqui. (Univ. Fed. } \\
\text { Estado Rio J., Online) }\end{array}$ \\
\hline $\begin{array}{l}\text { A dor no puerpério imediato: contribuição do } \\
\text { cuidado de enfermagem }\end{array}$ & 2018 & Figueiredo, J. V et al & Rev. bras. enfermagem \\
\hline $\begin{array}{c}\text { Atuação do enfermeiro na visita domiciliar } \\
\text { puerperal: perspectivas sobre o papel } \\
\text { profissional }\end{array}$ & 2019 & Ferreira, J. A. R.et al & Rev. baiana saúde pública \\
\hline $\begin{array}{l}\text { Assistência puerperal e a construção de um } \\
\text { fluxograma para consulta de enfermagem }\end{array}$ & 2020 & Silva, L. P. et al & $\begin{array}{l}\text { Rev. Bras. Saúde Mater. } \\
\text { Infant. (Online) }\end{array}$ \\
\hline $\begin{array}{l}\text { Gestão do cuidado de enfermagem a } \\
\text { puérperas e recém-nascidos na Atenção } \\
\text { Primária à Saúde }\end{array}$ & 2020 & Amorim, T. S.et al & Rev Rene (online) \\
\hline
\end{tabular}

Fonte: Autores.

Após a análise e classificação das informações coletadas, acerca da assistência de enfermagem à mulher no puerpério foram construídas 2 categorias. Categoria 1: A assistência de enfermagem no puerpério e Categoria 2- A percepção da puérpera sobre o cuidado de enfermagem. 


\section{Discussão}

\section{Categoria 1: A assistência de enfermagem no puerpério}

Prigol e Baruffi (2017) evidenciaram em seu estudo os cuidados de enfermagem tanto a puérpera quanto ao RN, destacando-se os cuidados de enfermagem em ambiente hospitalar e os cuidados na ESF, como orientações relacionadas à puericultura, amamentação, escuta ativa, cuidados dirigidos às alterações fisiológicas referentes ao puerpério, cuidados com o pós-parto, visita domiciliar, realizando o seu papel como educador e profissional na proteção e promoção a saúde.

A atuação da equipe de enfermagem é de grande importância na assistência puerperal. Acredita-se que deve se valorizar a individualidade de cada puérpera para que possa ser ofertado um atendimento humanizado e seguro. Visando um atendimento integral os cuidados devem ser direcionados a prevenção das complicações que possam surgir durante este período, dando conforto físico e emocional (Strefling et al., 2017).

No estudo de Dantas et al., (2018) evidenciou-se que o cuidado de enfermagem no puerpério teve foco os cuidados ao RN, onde as mães só receberam orientações sobre maternidade, mas priorizando os cuidados sobre amamentação. As orientações sobre os cuidados com o RN, amamentação e o autocuidado no puerpério são de suma importância na nova experiência não só da puérpera como da família, mas é preciso que essas mulheres recebam informações sobre autocuidado com elas mesmas e com o RN, para que se sintam confiantes, e assim aja uma maior interação entre cliente e profissional. Já muitas vezes as puérperas tem sentimentos negativos como o medo, insegurança e angústia em relação a essa nova fase da sua vida, isso tende a ser mais comum em mães primíparas, porém mães que tem mais de um filho também podem se sentir assim (Mercado et al., 2017).

Figueiredo et al., (2018) através da pesquisa foi possível evidenciar as contribuições da assistência de enfermagem no que diz respeito ao conforto da mulher em puerpério imediato, através de orientações sobre a relação da cólica com a amamentação, fisiologia do puerpério, posição e pega correta do RN, prevenção e tratamento das fissuras no mamilo e ingurgitamento.

O enfermeiro tem papel importante sobre o processo de aleitar, dando orientações oportunas sobre posicionamento mãe e bebê, pega correta, fissuras, ingurgitamento, e até mesmo e não menos importante que as orientações práticas sobre os cuidados com a própria mulher em puerpério (Galvão \& Silva, 2013).

Ferreira et al., (2019) explicaram que durante a visita domiciliar as orientações que foram passadas pela enfermeira em sua maioria eram sobre amamentação, sendo assim outros cuidados sobre o RN e a puérpera não foram abordadas de forma completa, as participantes da pesquisa relataram que a atuação do enfermeiro referente às orientações durante a visita puerperal deve ser mais completa, para que este profissional possa abordar todos os cuidados possíveis, principalmente os que possam trazer alguma intercorrência por falta de orientações.

Francisquini et al., (2010) constataram que para uma adequada assistência de enfermagem a puérpera, é necessário que se tenha uma adequação as especificidades deste período na vida da mulher, e o diálogo entre o profissional enfermeiro e a puérpera. Tendo que o enfermeiro oferecer informações, acolhendo as crenças, vivências e, procurando então desmistificar o que for negativo para esta mulher. A puérpera que tem informações e orientações durante o pré-natal referentes à gestação atravessará esta fase com uma maior segurança, prazer e harmonia, ao contrário das que não buscam ou não recebem estas informações, passaram por esta fase com preocupações desnecessárias e expectativas frustrantes.

Amorim et al., (2020) explicam que a gestão do cuidado de enfermagem a puérperas e recém-nascidos na Atenção Primária à Saúde (APS) significa acolher as singularidades de e mãe-filho e da família do mesmo, desde o início do pré-natal, promovendo uma assistência singular. Tornando a puérpera protagonista dos cuidados consigo e com o recém-nascido, além de envolver a participação e o apoio da família nos cuidados. 
Para desenvolver o cuidado puerperal, o enfermeiro deve estar preparado para auxiliar na transição de gestante para puérpera, conhecer a mulher durante este período, para que possa proporcionar a troca de informações sobre a sua saúde e seus direitos, favorecendo com isso, a autonomia e garantindo a sua participação na tomada de decisões (Souza et al., 2017; Araújo \& Mandúent, 2015).

Diante disso, a informação sobre consulta puerperal é importante e deve ser destacada durante o pré-natal. Sendo assim, a consulta puerperal permite a realização de ações de autocuida do e cuidados com o RN, orientações sobre amamentação, saúde sexual e reprodutiva, bem como a verificação das condições físicas, sociais, emocionais, além da vinculação que com o RN (Brasil, 2012).

Silva et al., (2020) realizaram um estudo na construção de um fluxograma para atendimento de enfermagem nas consultas puerperais em um hospital privado referência em São Paulo, onde observou-se que os problemas e queixa mais comuns evidenciados pelas participantes foi o cuidado com o RN, aleitamento materno, estado emocional e o suporte familiar. O Enfermeiro deve considerar o meio sociocultural onde está puérpera está inserida, com o objetivo de atrelar suas crenças com a ciência, para que se tenham um melhor resultado quanto às orientações, dessa forma é fundamental que o enfermeiro esteja atento á essas necessidades apresentadas, atuando com foco na escuta dessas puérperas, lhes oferecendo apoio e promovendo intervenções para ajudá-las em suas dificuldades (Ribeiro, 2021).

\section{Categoria 2: A percepção da puérpera sobre o cuidado de enfermagem}

Streflinet al., (2017) evidenciaram em seu estudo que a percepção da maioria das puérperas foi positiva em relação aos cuidados de enfermagem, e reconheceram a importância da relação profissional-cliente durante sua hospitalização. Porém ao conhecer a percepção de puérperas adolescentes do alojamento conjunto foi apontada a falta de diálogo, ajuda e presença do enfermeiro.

Segundo Rogerio et al., (2020) O contato mais próximo promovido pelo alojamento conjunto permite que a equipe de saúde, em especial a equipe de enfermagem, promova o apoio e orientação materna acercados cuidados essenciais para si e seu bebê. O profissional de saúde possui a responsabilidade de transferir conhecimento e fornecer orientações, segundo o Ministério da Saúde, ao orientar a mãe quanto a maneira correta de realizar o banho e os cuidados, estes promovem conforto, higiene e interação mãe-filho.

Silva et al.,(2017)observaram em sua pesquisa que a assistência de enfermagem ficou limitada às orientações no momento da alta hospitalar onde se percebeu através das falas das puérperas as lacunas quanto ao seu alto cuidado e o cuidado ao RN. Quanto as visitas domiciliares foram evidenciadas satisfação pela assistência de enfermagem com tudo foi evidente as lacunas. Lima e Araújo (2021) explicam que a visita domiciliar tem um significado relevante na vida das usuárias, fato constatado quando as mulheres relatam que ficaram muito satisfeitas com a visita do enfermeiro, mesmo com assistência fragmentada. Quando organizada e planejada a visita torna-se uma importante ferramenta para a construção de vínculos e fundamental para a promoção da saúde, principalmente no período puerperal, pois uma orientação realizada dentro de um domicílio permite ao profissional analisar vários contextos e intervir da melhor forma possível.

Ebling et al., (2018) evidenciaram em seu estudo um cuidado tecnicista, fragmentado e desprovido de uma assistência integral e focada no RN, porem foi demonstrado na fala das participantes uma assistência atenciosa. Reichert et al., (2016) constatou que os cuidados prestados pelo profissional enfermeiro são muito importantes neste período, porém de forma alguma podem ser limitados ou fragmentados, tendo em vista que a puérpera e o RN estão vulneráveis a várias enfermidades nesse período. Portanto, a atuação do enfermeiro deve ser mais abrangente do que apenas a orientação quanto ao uso do sulfato ferroso para a mãe e a importância do leite materno para o filho. 
Acredita-se que esta revisão possa contribuir para uma assistência de enfermagem com qualidade, fazendo com que estes profissionais aprimorem seu trabalho e potencialize a qualidade na assistência prestada a mulher em período puerperal, fazendo com que reflitam sobre seu fazer e sentir, possibilitando contribuir de forma segura nesta fase de mudanças. Podendo ainda enfatizar que as ações de saúde, ou a falta delas, podem refletem de forma direta e indireta na saúde da puérpera, do seu bebê e de toda a família.

Reconhecem-se as fragilidades do estudo e recomenda-se o aprofundamento do mesmo para um maior conhecimento teórico-prático e de novas pesquisas científicas para ampliação, divulgação e desenvolvimento de melhores práticas assistenciais às puérperas.

\section{Considerações Finais}

Através desta revisão foi possível evidenciar que o puerpério é um período de muitas mudanças físicas e emocionais onde a mulher vivência uma nova fase de sua vida, e a enfermagem tem um papel importante neste período. Porém foi possível observar que ainda faltam cuidados de enfermagem qualificados com base na prevenção de complicações que possam surgir neste período, o conforto e ajuda emocional e educação em saúde, onde o Enfermeiro exerça seu papel como educador.

É necessário destacar que a assistência no puerpério vem deixando a desejar no que diz respeito a educação em saúde, onde mesmo em ambiente hospitalar e fora dele as mulheres continuam com muitas dúvidas, quanto aos cuidados consigo mesma, com o RN, planejamento familiar e aleitamento materno.

É preciso que este profissional veja a importância desta fase para que possam elucidar as lacunas para uma melhor qualidade de vida das puérperas principalmente no alto cuidado delas, pois foi observado que á um maior privilégio de cuidados e orientações referentes ao recém-nascido, fazendo com que as orientações e cuidados com a puérpera fiquem de lado. A assistência de enfermagem precisa ir além a suas orientações e cuidados, tanto no ambiente hospitalar durante a alta quanto em visitas domiciliares.

Diante do exposto acredita-se que a enfermagem deva estar constantemente se aprimorando quanto aos seus conhecimentos para uma assistência que possa atender as demais demandas e singularidades de cada puérpera. Pois o puerpério é uma fase com possibilidade de riscos e agravos, onde os cuidados devem visar a prevenção de complicações que podem repercutir tanto na mãe como no recém-nascido e família.

\section{Referências}

Amorim, T. S., \&Backes, M. T. S. (2020). Gestão do cuidado de enfermagem a puérperas e recém-nascidos na Atenção Primária à Saúde, 21, e43654

Araujo, N. B. D., \&Mandú, E. N. T. (2016). Produção de sentidos entre adolescentes sobre o cuidado de si na gravidez. Interface-Comunicação, Saúde, Educação, 20, 363-375.

Brasil. (2011) Ministério da Saúde. Manual prático para implementação da rede da http://www.saude.ba.gov.br/wpcontent/uploads/2017/09/MANUAL_PRATICO_MS_IMPLEMENTACAI_REDE_CEGONHA.pdf.

Brasil.Secretaria de Atenção à Saúde. Departamento de Atenção Básica. Atenção a natal.https://bvsms.saude.gov.br/bvs/publicacoes/cadernos_atencao_basica_32_prenatal.pdf>.

Cunha, M. R., et al. (2018). Identificação da infecção de sítio cirúrgico pós-cesariana: consulta de enfermagem. Revista Brasileira de Enfermagem, 71, 13951403 .

Dantas, S. L. C., et al. (2018). Representações sociais de enfermeiros da atenção primária à saúde sobre cuidado de enfermagem no pós-parto. Cogitare enfermagem, 23(3).

Dyniewicz, A. M. (2014). Metodologia da Pesquisa em Saúde para Iniciantes. rev. ampl. São Caetano do Sul, SP. Difusão Editora.

Lima, C. S., \& de Araújo, T. C. V. (2021). A visita domiciliar do enfermeiro da estratégia saúde da família na atenção ao puerpério. Revista Ciência Plural, 7(3), 314-331.

Ebling, S. B. D., et al. (2018). Compreensões de cuidado na visão de mulheres puérperas. Revista Online de Pesquisa Cuidado é Fundamental, $10(1)$, $30-35$. 
Ferreira, J. A. R., et al. (2019). Atuação do enfermeiro na visita domiciliar puerperal: perspectivas sobre o papel profissional. Revista Baiana de Saúde Pública, 43(3), 567-580.

Figueiredo, J. V., et al. (2018). El dolor em el puerperio in mediato: contribución del cuidado de enfermeira. Revista Brasileira de Enfermagem, 71, 13431350 .

Francisquini, A. R., et al. (2010). Orientações recebidas durante a gestação, parto e pós-parto por um grupo de puérperas. Ciência, Cuidado e Saúde, 9(4), 743751.

Galvão, D. M. P. G., \& Silva, I. A. (2013). Abordagem da amamentação nos primeiros anos do ensino fundamental. Revista da Escola de Enfermagem da USP, 47, 477-485.

Gomes, G. F., \& Dos Santos, A. P. V. (2017). Assistência de enfermagem no puerpério. Revista Enfermagem Contemporânea, 6(2), 211-220.

Gonçalves, M. V. C., et al.(2012) Endometrite puerperal e sepse: Uma revisão. Rev. Med. Minas Gerais, 22, S21-S24.

Martins, S. V., et al. (2012) Ações de enfermagem no período puerperal na atenção primaria a saúde. Rev. Tendência da Enfermagem Profissional, 4 (4), 833838 .

Mendes, K. D. S., Silveira, R. C. D. C. P., \& Galvão, C. M. (2008). Revisão integrativa: método de pesquisa para a incorporação de evidências na saúde e na enfermagem. Texto \& contexto-enfermagem, 17, 758-764.

Mercado, N. C., et al. (2017). Cuidados e orientações de enfermagem às puérperas no alojamento conjunto. Rev. enferm. UFPE on line, 3508-3515.

Prigol, A. P., \&Baruffi, L. M. (2017). O papel do enfermeiro no cuidado à puérpera. Rev. enferm. UFSM, 1-8.

Quiros, C. C., \& Barboza, L. S. Propuesta para la creacíón de una consulta postparto dada por Enfermería Ginecológica Obstétrica y Perinatal enel Área de Salud de Coronado. Enfermería Actualen Costa Rica, (31), 1-19.

Reichert, A. P. S., et al. (2016). Primeira Semana Saúde Integral: ações dos profissionais de saúde na visita domiciliar ao binômio mãe-bebê. Revista Enfermagem UERJ, 24 (5), 27955.

Ribeiro, T. P. (2021). Percepção de enfermeiras de unidades básicas de saúde acerca das alterações emocionais no puerpério.

Rogerio, M. C., et al. (2020). Orientações para puérperas sobre cuidados neonatais no alojamento conjunto em maternidades de risco habitual. Enfermagem em Foco, 11(1).

Silva, E. D. C., et al. (2017). Puerpério e assistência de enfermagem: percepção das mulheres. Rev. enferm. UFPE online, $2826-2833$.

Silva, L. P. D., et al. (2020). Assistência puerperal e a construção de um fluxograma para consulta de enfermagem. Revista Brasileira de Saúde Materno Infantil, 20, 101-113.

Soares, C. (2007). Assistência de enfermagem no puerpério em unidade de atenção básica: incentivando o autocuidado.

Souza, A. B. Q., \& Fernandes, B. M. (2014). Diretriz para assistência de enfermagem: ferramenta eficaz para a promoção da saúde no puerpério. Rev Rene, 15(4), 594-604.

Souza, M. G., de Oliveira, C. A., \&Justi, J. (2017). Puerpério e atenção à saúde: percepção de mulheres assistidas pelo Sistema Único de Saúde. Revista Saúde e Desenvolvimento, 11(7), 158-177.

Souza, M. T., et al. (2010). Revisão integrativa: o que é e como fazer. Einsten (São Paulo), 8 (1), 102-106.

Strefling, I. D. S. S., et al (2017). Percepções de puérperas sobre o cuidado de enfermagem no alojamento conjunto. Revista de Pesquisa: Cuidado é fundamental online, 9(2), 333-339. 